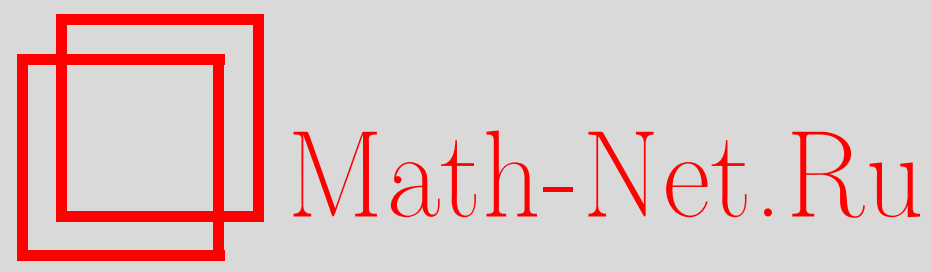

Н. Т. Лупашко, Н. И. Санду, О полугруппах эндоморфизмов прямых произведений коммутативных луп Муфанг, Дискрет. матем., 2011, том 23, выпуск 1, 84-93

DOI: https://doi.org/10.4213/dm1132

Использование Общероссийского математического портала Math-Net.Ru подразумевает, что вы прочитали и согласны с пользовательским соглашением http://www . mathnet.ru/rus/agreement

Параметры загрузки:

IP: 3.89 .185 .249

26 апреля 2023 г., 15:32:11 


\title{
О полугруппах эндоморфизмов прямых произведений коммутативных луп Муфанг
}

\author{
(c) 2011 г. Н. Т. Лупашко, Н. И. Санду
}

\begin{abstract}
Доказывается, что полугруппа эндоморфизмов коммутативной лупы Муфанг КЛМ, обладающей разложением в прямое произведение своих подлуп, изоморфна полугруппе $M, M$-матриц. Вводится понятие голоморфа КЛМ и для КЛМ, обладающих прямым разложением, приводится матричное представление голоморфа.
\end{abstract}

Известно, что эндоморфизмы и автоморфизмы векторных пространств могут быть представлены матрицами над соответствующими полями. Это матричное представление играет большую роль в теории линейных групп - групп автоморфизмов векторных пространств. Аналогичное матричное представление рассматривается и для прямых произведений мультиоператорных групп [1]. В данной работе этот вопрос рассматривается для наиболее изученного класса неассоциативных луп - коммутативных луп Муфанг (кратко КЛМ). Доказывается, что полугруппа эндоморфизмов КЛМ, обладающая разложением в прямое произведение своих подлуп, изоморфна полугруппе $M, M$-матриц. Имеются и другие сведения об эндоморфизмах и автоморфизмах прямых разложений КЛМ. В частности, вводится понятие голоморфа КЛМ и приводится матричное представление голоморфа КЛМ, обладающих разложением в прямое произведение своих подлуп.

Необходимые сведения по теории луп можно найти в [2]. Напомним только, что группа внутренних подстановок произвольной лупы порождается подстановками

$$
\begin{gathered}
T(a)=L^{-1}(a) R(a), \quad L(a, b)=L^{-1}(a, b) L(a) L(b), \\
R(a, b)=R^{-1}(a, b) R(b) R(a),
\end{gathered}
$$

где $L(a) x=a x, R(a) x=x a$. В КЛМ внутренние подстановки являются ее автоморфизмами.

Пусть КЛМ $Q$ разлагается в прямое произведение своих компонент

$$
Q=Q_{1} \times Q_{2} \times \ldots \times Q_{r} \times \ldots
$$

Если $u \in Q_{i}, v \in Q_{j}, w \in Q_{t}$ и по крайней мере два из трех индексов $i, j, t$ различны, то из определения прямого произведения следует, что $u v \cdot w=u \cdot v w$. Более того, рассмотрим произведение $a=\left(a_{1} a_{2} \cdots a_{n}\right)_{\alpha}$ при некотором распределении скобок $\alpha$, где $a_{i} \in Q_{j}$. Если $\left(a_{1} a_{2} \cdots a_{n}\right)_{\alpha}$ содержит не более двух сомножителей $a_{i}, a_{j}, i, j=1, \ldots, n$, принадлежащих одной и той же компоненте $Q_{k}$, то значение выражения $\left(a_{1} a_{2} \cdots a_{n}\right)_{\alpha}$ не меняется при любом другом распределение скобок $\alpha$ и при любой перестановке сомножителей $a_{1}, a_{2}, \ldots, a_{n}$. Такое свойство выражения $a$ назовем компонентно ассоциативным. 
Доказательство этого утверждения проведем индукцией по длине $n$ слова $a$. Его справедливость для $n=3$ указана выше. Пусть $n>3$ и $a=u v$. Предположим, что $u$ содержит сомножитель $a_{k}$. Тогда по индуктивному предположению $u=u_{1} a_{k}$. Утверждение будет доказано, если мы покажем, что $a=u_{1} a_{k} \cdot v=u_{1} \cdot a_{k} v$ или $\left(u_{1}, a_{k}, v\right)=1$, где $x y \cdot z=(x \cdot y z)(x, y, z)$. Предположим, что $u_{1}=u_{2} u_{3}$. Используем тождество

$$
(x y, z, t)=(x, z, t)((x, z, t), x, y) \cdot(y, z, t)((y, z, t), y, x),
$$

справедливое в произвольной КЛМ. Тогда

$$
\begin{aligned}
\left(u_{1}, a_{k}, v\right) & =\left(u_{2} u_{3}, a_{k}, v\right) \\
& =\left(u_{2}, a_{k}, v\right)\left(\left(u_{2}, a_{k}, v\right), u_{2}, u_{3}\right) \cdot\left(u_{3}, a_{k}, v\right)\left(\left(u_{3}, a_{k}, v\right), u_{3}, u_{2}\right)=1,
\end{aligned}
$$

поскольку по индуктивному предположению $\left(u_{2}, a_{k}, v\right)=1,\left(u_{3}, a_{k}, v\right)=1$. Если же $v=v_{1} v_{2}$, то достаточно воспользоваться тождеством $(x, y, z)=(y, z, x)$ (см. [2]). Этим показано, что КЛМ $Q$ является компонентно ассоциативной.

Ввиду разложения (1) каждый элемент $a \in Q$ имеет вид $a=\prod_{i=1}^{t} a_{i}$. Если $\varphi-$ эндоморфизм КЛМ $Q$, то $\varphi a=\prod_{i=1}^{t} \varphi a_{i}$. Поэтому для определения эндоморфизма $\varphi$ достаточно указать, как действует $\varphi$ на компонентах $Q_{i}$. Если $\varphi a_{i}=\prod a_{k i}$, то будем писать $\varphi a_{i}=\prod \varphi_{k i} a_{i}$. Так как элемент $a_{k i}=\varphi_{k i} a_{i}$ однозначно определяется элементом $a_{i}$ и отображением $\varphi$, то $\varphi_{k i}$ дает однозначное отображение $Q_{i}$ в $Q_{k}$. Покажем, что $\varphi_{i k}$ является гомоморфизмом КЛМ $Q_{i}$ в КЛМ $Q_{k}$. Действительно, пусть $a_{i}, b_{i} \in Q_{i}$ и

$$
\begin{aligned}
\varphi a_{i} & =\varphi_{1 i} a_{i} \cdots \varphi_{k i} a_{i} \cdots \varphi_{n i} a_{i}, \\
\varphi b_{i} & =\varphi_{1 i} b_{i} \cdots \varphi_{k i} b_{i} \cdots \varphi_{n i} b_{i} .
\end{aligned}
$$

Ввиду компонентной ассоциативности КЛМ $Q$ относительно подлуп $Q_{i}$, в последних двух выражениях скобки опускаем. Тогда

$$
\begin{aligned}
\varphi a_{i} \varphi b_{i} & =\left(\varphi_{1 i} a_{i} \cdots \varphi_{k i} a_{i} \cdots \varphi_{n i} a_{i}\right)\left(\varphi_{1 i} b_{i} \cdots \varphi_{k i} b_{i} \cdots \varphi_{n i} b_{i}\right) \\
& =\varphi_{1 i} a_{i} \varphi_{1 i} b_{i} \cdots \varphi_{k i} a_{i} \varphi_{k i} b_{i} \cdots \varphi_{n i} a_{i} \varphi_{n i} b_{i} .
\end{aligned}
$$

Так как $\varphi-$ эндоморфизм КЛМ $Q$, то

$$
\varphi a_{i} \cdot \varphi b_{i}=\varphi\left(a_{i} b_{i}\right)
$$

Пусть

$$
\varphi\left(a_{i} b_{i}\right)=\varphi_{1 i}\left(a_{i} b_{i}\right) \cdots \varphi_{k i}\left(a_{i} b_{i}\right) \cdots \varphi_{n i}\left(a_{i} b_{i}\right)
$$

Тогда

$$
\varphi_{1 i} a_{i} \varphi_{1 i} b_{i} \cdots \varphi_{k i} a_{i} \varphi_{k i} b_{i} \cdots \varphi_{n i} a_{i} \varphi_{n i} b_{i}=\varphi_{1 i}\left(a_{i} b_{i}\right) \cdots \varphi_{k i}\left(a_{i} b_{i}\right) \cdots \varphi_{n i}\left(a_{i} b_{i}\right) .
$$

В этом соотношении только сомножители $\varphi_{k i} a_{i} \varphi_{k i} b_{i}$ и $\varphi_{k i}\left(a_{i} b_{i}\right)$ принадлежат компоненте $Q_{k}$. Тогда из определения прямого произведения следует, что

$$
\varphi_{k i} a_{i} \varphi_{k i} b_{i}=\varphi_{k i}\left(a_{i} b_{i}\right)
$$

Следовательно, $\varphi_{k i}$ является гомоморфизмом КЛМ $Q_{i}$ в КЛМ $Q_{k}$.

Лемма 1. Пусть КЛМ $Q$ разлагается в прямое произведение (1) и пусть $\varphi$ - эндоморфизм КЛМ $Q$. Тогда КЛМ $Q_{i}$ является компонентно ассоииативной относительно своих подлуп $\varphi_{i k} Q_{k}$. 
Доказательство. Пусть $a_{i} \in Q_{i}, b_{j} \in Q_{j}, c_{t} \in Q_{t}$, и пусть по крайней мере два из индексов $i, j, t$ различны. Тогда

$$
\begin{aligned}
a_{i} b_{j} \cdot c_{t} & =a_{i} \cdot b_{j} c_{t}, \\
\varphi\left(a_{i} b_{j} \cdot c_{t}\right) & =\varphi\left(a_{i} \cdot b_{j} c_{t}\right), \\
\varphi a_{i} \varphi b_{j} \cdot \varphi c_{t} & =\varphi a_{i} \cdot \varphi b_{j} \varphi c_{t} .
\end{aligned}
$$

Следовательно, справедливо равенство

$$
\prod \varphi_{k i} a_{i} \prod \varphi_{k j} b_{j} \cdot \prod \varphi_{k t} c_{t}=\prod \varphi_{k i} a_{i} \cdot \prod \varphi_{k j} b_{j} \prod \varphi_{k t} c_{t}
$$

Используя дальше компонентную ассоциативность КЛМ $Q$ относительно разложения (1) получим, что

$$
\begin{aligned}
\left(\varphi_{1 i} a_{i} \varphi_{1 j} b_{j} \cdot \varphi_{1 t} c_{t}\right)\left(\prod_{k=2} \varphi_{k i} a_{i} \prod_{k=2} \varphi_{k j} b_{j} \cdot \prod_{k=2} \varphi_{k t} c_{t}\right) \\
=\left(\varphi_{1 i} a_{i} \cdot \varphi_{1 j} b_{j} \varphi_{1 t} c_{t}\right)\left(\prod_{k=2} \varphi_{k i} a_{i} \cdot \prod_{k=2} \varphi_{k j} b_{j} \prod_{k=2} \varphi_{k t} c_{t}\right) .
\end{aligned}
$$

Отсюда следует, что

$$
\varphi_{1 i} a_{i} \varphi_{1 j} b_{j} \cdot \varphi_{1 t} c_{t}=\varphi_{1 i} a_{i} \cdot \varphi_{1 j} b_{j} \varphi_{1 t} c_{t} .
$$

Аналогично проверяются равенства

$$
\varphi_{k i} a_{i} \varphi_{k j} b_{j} \cdot \varphi_{k t} c_{t}=\varphi_{k i} a_{i} \cdot \varphi_{k j} b_{j} \varphi_{k t} c_{t}, \quad k=2,3, \ldots
$$

Рассмотрим теперь произведение $d=d_{1} d_{2} \cdots d_{n}$ при некотором распределении скобок, где $d_{i} \in \varphi_{k j} Q_{j}, d_{i}=\varphi_{k j} a_{j}$, и при условии, что в слове $d$ встречаются не более двух переменных, принадлежащих одной и той же подлупе $\varphi_{k j} Q_{j} \subseteq Q_{k}$. Пусть $a=a_{1} a_{2} \cdots a_{n}$. Тогда $\varphi a=d$. Элемент $a$ компонентно ассоциативен относительно сомножителей $a_{i}$. Отсюда следует, что и элемент $d$ компонентно ассоциативен относительно сомножителей $d_{i} \in \varphi_{k j} Q_{j}$. Этим доказательство леммы завершается.

Теперь, по аналогии с [1], введем следующие понятия. Пусть $M, N-$ два множества и $L-$ алгебраическая система с частичными операциями умножения ·, сложения + и нульарной операцией 0, свойства которых будут указаны в конкретных примеpax. Будем рассматривать всевозможные функции $A, B, \ldots$ двух переменных, определенные на декартовом произведении $A \times B$ и со значениями в $L$. Такие функции назовем $M, N$-матрицами над $L$. Через $a_{\alpha \beta}$ будем обозначать значения функции при аргументах $\alpha$ и $\beta: A(\alpha, \beta)=a_{\alpha \beta}$, а саму функцию-матрицу обозначим через $\left(a_{\alpha \beta}\right)$. Если $A=\left(a_{\alpha \beta}\right)$ - матрица, то, фиксируя в ней первый аргумент, мы получим функцию одной переменной - строку $\bar{a}_{\alpha}=\left(\bar{a}_{\alpha}(\beta)\right)$, и аналогично, фиксируя второй аргумент, получим столбец $\bar{a}^{\beta}=\left(\bar{a}^{\beta}(\alpha)\right)$. Матрицу $A$ назовем конечнострочной, если во всех ее строках имеется лишь конечное число ненулевых значений. Соответственно определяются матрицы с конечными столбцами. Пусть теперь $A-M, N$-матрица и $B-N, K$-матрица, причем либо $A-$ конечнострочная, либо $B-$ конечностолбцовая. Тогда их произведение $C=A B-$ $M, K$-матрица, определяемая формулой

$$
c_{\alpha \beta}=\sum_{\gamma} a_{\alpha \gamma} b_{\gamma \beta},
$$


где $\alpha \in M, \gamma \in N, \beta \in K$, и мы считаем, что, суммируя бесконечное число нулей, снова получим нуль. Наконец, функция $\bar{a}=\left(\bar{a}_{\alpha \alpha}\right)$ - это главная диагональ $M, M$-матрицы $\left(a_{\alpha \beta}\right)$.

Теперь переходим к исследованию связей между $M, M$-матрицами и эндоморфизмами КЛМ $Q$, обладающей разложением в прямое произведение (1). Как указано перед леммой 1 , любому эндоморфизму $\varphi$ КЛМ $Q$ можно поставить в соответствии конечнострочную и конечностолбцовую $M, M$-матрицу из гомоморфизмов $\varphi_{k i}: Q_{i} \rightarrow Q_{k}$. Если разложение (1) содержит $n$ сомножителей, то матрица $\left(\varphi_{k i}\right)$, соответствующая эндоморфизму $\varphi$, состоит из $n^{2}$ гомоморфизмов $\varphi_{k i}$.

Докажем обратное утверждение, что $M, M$-матрица, состоящая из гомоморфизмов $\varphi_{k i}: Q_{i} \rightarrow Q_{k}$ и удовлетворяющая условиям леммы 1 , однозначно определяет эндоморфизм $\varphi$ КЛМ $Q$. Действительно, пусть $a, b-$ произвольные элементы из $Q$ и пусть

$$
a=\prod_{i=1}^{k} a_{i}, \quad b=\prod_{i=1}^{k} b_{i}, \quad a_{i}=\prod_{j=1}^{t} a_{j i}, \quad b_{i}=\prod_{j=1}^{t} b_{j i},
$$

где $a_{i}, b_{i} \in Q_{i}, a_{j i}, b_{j i} \in Q_{j}$. При помощи заданных гомоморфизмов $\varphi_{j i}: Q_{i} \rightarrow Q_{j}$ определим отображение $\psi$ множества $Q$, полагая

$$
\psi a_{i}=\prod_{j=1}^{t} \varphi_{j i} a_{i}, \quad \psi b_{i}=\prod_{j=1}^{t} \varphi_{j i} b_{i}, \quad \mu a=\prod_{i=1}^{k} \psi a_{i}, \quad \mu b=\prod_{i=1}^{k} \psi b_{i} .
$$

Отметим, что при переходе к матричному изображению эндоморфизма $\varphi$ определение гомоморфизмов $\varphi_{j i}$ зависит от элементов подлуп $Q_{i}$ и $Q_{j}$. Покажем, что отображение $\mu$ является эндоморфизмом КЛМ $Q$. Пусть еще

$$
a b=\prod_{i=1}^{k}(a b)_{i}, \quad(a b)_{i}=\prod_{j=1}^{t} \varphi_{j i}(a b)_{i} .
$$

Тогда

$$
\mu(a b)=\prod_{i=1}^{k}\left(\prod_{j=1}^{t} \varphi_{j i}(a b)_{i}\right)
$$

Преобразуем $\psi(a b)$. Для этого будем использовать соотношение

$$
\varphi_{j i}(a b)_{i}=\varphi_{j i}\left(a_{i} b_{i}\right)=\varphi_{j i} a_{i} \varphi_{j i} b_{i}
$$

и компонентную ассоциативность КЛМ $Q_{j}$ относительно $\varphi_{j i} Q_{i}$. Из этого соотношения следует, что $\psi$ является эндоморфизмом подлуп $Q_{i}$. После будем использовать компонентную ассоциативность КЛМ $Q$ относительно подлуп $Q_{i}$. Справедливы равенства

$$
\begin{aligned}
\mu(a b) & =\prod_{i=1}^{k} \psi(a b)_{i}=\prod_{i=1}^{k}\left(\prod_{j=1}^{t} \varphi_{j i}(a b)_{i}\right) \\
& =\prod_{i=1}^{k}\left(\prod_{j=1}^{t}\left(\varphi_{j i} a_{i} \cdot \varphi_{j i} b_{i}\right)\right)=\prod_{i=1}^{k}\left(\prod_{j=1}^{t} \varphi_{j i} a_{i} \cdot \prod_{j=1}^{t} \varphi_{j i} b_{i}\right) \\
& =\prod_{i=1}^{k}\left(\psi a_{i} \cdot \psi b_{i}\right)=\prod_{i=1}^{k} \psi a_{i} \cdot \prod_{i=1}^{k} \psi b_{i}=\mu a \cdot \mu b .
\end{aligned}
$$


Следовательно, $\mu$ - эндоморфизм КЛМ $Q$, который индуцирует те же гомоморфизмы между подлупами $Q_{i}, Q_{j}$, что и $\varphi$. Тогда эндоморфизмы $\mu$ и $\varphi$ совпадают. Этим показано, что между эндоморфизмами КЛМ $Q$ и $M, M$-матрицами с условием компонентной ассоциативности существует взаимно однозначное соответствие.

Найдем далее матрицу, отвечающую произведению эндоморфизмов. Для этого предварительно покажем, как применение эндоморфизма к элементам КЛМ $Q$ с разложением (1) сводится к матричному умножению. Пусть $a \in Q$ и $\varphi-$ эндоморфизм КЛМ $Q$. Согласно (1), $a=\prod a_{i}$, где $a_{i} \in Q_{i}, i=1,2, \ldots, r, \ldots$, и только конечное число сомножителей $a_{i}$ отличны от единицы. Через $\bar{a}$ обозначим матрицу-столбец с элементами $a_{1}, a_{2}, \ldots, a_{r}, \ldots$, а через $\bar{\varphi}=\left(\varphi_{k j}\right)-M, M$-матрицу, соответствующую эндоморфизму $\varphi$. Тогда $\bar{\varphi} \bar{a}=\bar{\varphi} \cdot \bar{a}$, где справа матрица умножается на столбец и в результате получается столбец.

В дальнейшем для компонентно ассоциативного выражения $a=a_{1} a_{2} \cdots a_{n}$ будем использовать и аддитивную запись $a=a_{1}+a_{2}+\ldots+a_{n}$. Тогда

$$
(\varphi a)=\sum_{j}\left(\sum_{k} \varphi_{j k} a_{k}\right)
$$

Покажем, что

$$
\sum_{j}\left(\sum_{k} \varphi_{j k} a_{k}\right)=\sum_{k}\left(\sum_{j} \varphi_{j k} a_{k}\right) .
$$

Будем использовать соотношение $\varphi_{j k} a_{k} \in Q_{j}$ для $a_{k} \in Q_{k}$, а также компонентную ассоциативность КЛМ $Q$ относительно своих подлуп $Q_{i}$ и компонентную ассоциативность КЛМ $Q_{j}$ относительно своих подлуп $\varphi_{j k} Q_{k}$. Справедливы равенства

$$
\begin{aligned}
\sum_{i=1}^{t} \sum_{k=1}^{t} \varphi_{j k} a_{k}=( & \left.\varphi_{11} a_{1}+\varphi_{12} a_{2}+\varphi_{13} a_{3}+\ldots+\varphi_{1 t} a_{t}\right) \\
& +\left(\varphi_{21} a_{1}+\varphi_{22} a_{2}+\varphi_{23} a_{3}+\ldots+\varphi_{2 t} a_{t}\right) \\
& +\left(\varphi_{31} a_{1}+\varphi_{32} a_{2}+\varphi_{33} a_{3}+\ldots+\varphi_{3 t} a_{t}\right)+\ldots \\
& +\left(\varphi_{t 1} a_{1}+\varphi_{t 2} a_{2}+\varphi_{t 3} a_{3}+\ldots+\varphi_{t t} a_{t}\right) \\
= & \left(\varphi_{11} a_{1}+\left(\varphi_{12} a_{2}+\varphi_{13} a_{3}+\ldots+\varphi_{1 t} a_{t}\right)\right) \\
& \left.+\left(\varphi_{21} a_{1}+\left(\varphi_{22} a_{2}+\ldots+\varphi_{2 t} a_{t}\right)\right)\right) \\
& +\left(\varphi_{31} a_{1}+\varphi_{32} a_{2}+\ldots+\varphi_{3 t} a_{t}\right)+\ldots \\
& +\left(\varphi_{t 1} a_{1}+\varphi_{t 2} a_{2}+\varphi_{t 3} a_{3}+\ldots+\varphi_{t t} a_{t}\right) \\
= & \varphi_{11} a_{1}+\varphi_{21} a_{1}+\left(\varphi_{12} a_{2}+\varphi_{13} a_{3}+\ldots+\varphi_{1 t} a_{t}\right) \\
& \left.+\left(\varphi_{22} a_{2}+\varphi_{23} a_{3}+\ldots+\varphi_{2 t} a_{t}\right)\right) \\
& +\left(\varphi_{31} a_{1}+\varphi_{32} a_{2}+\varphi_{33} a_{3}+\ldots+\varphi_{3 t} a_{t}\right)+\ldots \\
& +\left(\varphi_{t 1} a_{1}+\varphi_{t 2} a_{2}+\varphi_{t 3} a_{3}+\ldots+\varphi_{t t} a_{t}\right) \\
= & \left.\varphi_{11} a_{1}+\varphi_{21} a_{1}+\left(\varphi_{31} a_{1}+\left(\varphi_{32} a_{2}+\ldots+\varphi_{3 t} a_{t}\right)\right)\right)+\ldots \\
& +\left(\varphi_{t 1} a_{1}+\varphi_{t 2} a_{2}+\ldots+\varphi_{t t} a_{t}\right) \\
& +\left(\varphi_{12} a_{2}+\varphi_{13} a_{3}+\ldots+\varphi_{1 t} a_{t}\right) \\
& +\left(\varphi_{22} a_{2}+\varphi_{23} a_{3}+\ldots+\varphi_{2 t} a_{t}\right)
\end{aligned}
$$




$$
\begin{aligned}
& =\left(\varphi_{11} a_{1}+\varphi_{21} a_{1}+\varphi_{31} a_{1}\right)+\ldots \\
& \quad+\left(\varphi_{t 1} a_{1}+\varphi_{t 2} a_{2}+\varphi_{t 3} a_{3}+\ldots+\varphi_{t t} a_{t}\right) \\
& \quad+\left(\varphi_{12} a_{2}+\varphi_{13} a_{3}+\ldots+\varphi_{1 t} a_{t}\right) \\
& \quad+\left(\varphi_{22} a_{2}+\varphi_{23} a_{3}+\ldots+\varphi_{2 t} a_{t}\right) \\
& \quad+\left(\varphi_{32} a_{2}+\ldots+\varphi_{3 t} a_{t}\right)=\ldots \\
& =\left(\varphi_{11} a_{1}+\varphi_{21} a_{1}+\ldots+\varphi_{t 1} a_{1}\right) \\
& \quad+\left(\left(\varphi_{12} a_{2}+\varphi_{13} a_{3}+\ldots+\varphi_{1 t} a_{t}\right)\right. \\
& \quad+\left(\varphi_{22} a_{2}+\varphi_{23} a_{3}+\ldots+\varphi_{2 t} a_{t}\right) \\
& \quad+\left(\varphi_{32} a_{2}+\varphi_{33} a_{3}+\ldots+\varphi_{3 t} a_{t}\right)+\ldots \\
& \left.\quad+\left(\varphi_{t 2} a_{2}+\varphi_{t 3} a_{3}+\ldots+\varphi_{t t} a_{t}\right)\right)+\ldots \\
& =\left(\varphi_{11} a_{1}+\varphi_{21} a_{1} \ldots+\varphi_{t 1} a_{a}\right) \\
& \quad+\left(\left(\varphi_{12} a_{2}+\varphi_{22} a_{2}+\ldots+\varphi_{t 2} a_{2}\right)\right. \\
& \quad+\left(\left(\varphi_{13} a_{3}+\varphi_{23} a_{3}+\ldots+\varphi_{t 3} a_{3}\right)+\ldots\right. \\
& \left.\left.\quad+\left(\varphi_{1 t} a_{t}+\varphi_{2 t} a_{t}+\varphi_{3 t} a_{t}+\ldots+\varphi_{t t} a_{t}\right) \ldots\right)\right) \\
& =\sum_{k} \sum_{j} \varphi_{j k} a_{k} .
\end{aligned}
$$

Отметим, что из вышеизложенных преобразований следует, что суммирование по $k$ в последнем выражении производится при произвольном распределение скобок.

Совокупность всех эндоморфизмов произвольной алгебры естественным образом превращается в полугруппу. Умножением служит последовательное выполнение эндоморфизмов. Теперь найдем матрицу, отвечающую произведению эндоморфизмов КЛМ $Q$, обладающую разложением (1). Пусть $a \in Q, a=\prod a_{k}, a_{k} \in Q_{k}, \sigma$ и $\varphi-$ эндоморфизмы КЛМ $Q$, и пусть этим эндоморфизмам отвечают матрицы $\left(\sigma_{i j}\right)$ и $\left(\varphi_{j k}\right)$. Вычислим $\sigma(\varphi a)$, используя (2):

$$
\begin{aligned}
\sigma(\varphi a) & =\sigma\left(\sum_{j}\left(\sum_{k} \varphi_{j k} a_{k}\right)\right)=\sum_{j}\left(\sum_{k}\left(\sigma\left(\varphi_{j k} a_{k}\right)\right)\right) \\
& =\sum_{i}\left(\sum_{j}\left(\sum_{k}\left(\sigma_{i j}\left(\varphi_{j k} a_{k}\right)\right)\right)=\sum_{i}\left(\sum_{j}\left(\sum_{k}\left(\sigma_{i j}\left(\varphi_{j k} a_{k}\right)\right)\right)\right)\right. \\
& =\sum_{i}\left(\sum_{k}\left(\sum_{j}\left(\sigma_{i j}\left(\varphi_{j k} a_{k}\right)\right)\right)\right)=\sum_{i}\left(\sum_{k}\left((\sigma \varphi)_{i k} a_{k}\right)\right) .
\end{aligned}
$$

Заметим, что элементы матриц, соответствующих эндоморфизмам КЛМ $Q$, относятся к определенным разложениям (1). Элементы $\sigma_{i j} \varphi_{j k}$ являются гомоморфизмами из $Q_{k}$ в $Q_{i}$. Поэтому суммирование по $j$ в предыдущих выражениях следует понимать так:

$$
\left(\sigma_{i j_{1}} \varphi_{j_{1} k}+\sigma_{i j_{2}} \varphi_{j_{2} k}\right) a_{k}=\sigma_{i j_{1}} \varphi_{j_{1} k} a_{k} \cdot \sigma_{i j_{2}} \varphi_{j_{2} k} a_{k}
$$

Таким образом, доказано следующее утверждение.

Теорема 1. Полугруппа эндоморфизмов КЛМ Q, обладающая разложением в прямое произведение своих подлуп (1), изоморфна полугруппе $M, M$-матриц с условием компонентной ассоциативности, указанной в лемме 1. 
В [3] показано, что любая периодическая КЛМ разлагается в прямое произведение своих $p$-компонент, $p$-компоненты являются вполне характеристическими подлупами. Тогда из теоремы 1 вытекает следующее утверждение.

Следствие 1. Полугруппа эндоморфизмов периодической КЛМ изоморфна полугруппе диагональных $M, M$-матриц.

Как и для случая абелевых групп и их колец эндоморфизмов [4], справедливы следующие утверждения о взаимосвязи прямых разложений КЛМ $Q$ с их полугруппами эндоморфизмов $E(Q)$, а также с их $M, M$-матрицами, представляющими эти эндоморфизмы. Начнем со следующих утверждений.

(a) Если $Q=A \times B$, то полугруппу $E(B)$ можно рассматривать как подполугруппу полугруппь $E(Q)$, отождествив $E(B)$ с множеством всех $\alpha \in E(Q)$, которые индуцируют тождественное отображение на подлупу $A$. Более того, если $Q=\prod_{i \in I} A_{i}$, то декартово произведение $\bar{\prod}_{i \in I} E\left(A_{i}\right)$ всех полугрупп $E\left(A_{i}\right)$ является подполугруппой полугруппы $E(Q)$; эта подполугруппа состоит из всех эндоморфизмов $\alpha \in E(Q)$, которые каждую подлупу $A_{i}$ отображает на себя. При $M, M$-матричном представление эндоморфизмов $\alpha$ полугруппа $\bar{\prod}_{i \in I} E\left(A_{i}\right)$ - это в точности множество всех диагональных M, М-матриц вне диагонали которых стоят нули.

(b) Пусть $Q=B \times C$, и пусть $\varepsilon: Q \rightarrow B$ - соответствующая проекция. Тогда можно произвести отождествление

$$
E(B)=\varepsilon E(Q) \varepsilon .
$$

Если $\alpha \in E(Q)$, то $\varepsilon \alpha \varepsilon-$ эндоморфизм КЛМ $B$. С другой стороны, если $\theta-$ эндоморфизм КЛМ $B$, то, произведя указанное отождествление $\theta$ с эндоморфизмом КЛМ $Q$, получим, что $\theta=\varepsilon \theta \varepsilon$.

(c) Пусть КЛМ $Q=B \times C$ и $\bar{Q}$ имеют изоморфные полугруппь эндоморфизмов и пусть $\varphi: E(Q) \rightarrow E(\bar{Q})-$ изоморфизм между ними. Тогда $\bar{Q}=\bar{B} \times \bar{C}$, причем $\varphi$ индуцирует изоморфизмы $E(B) \rightarrow E(\bar{B})$ и $E(C) \rightarrow E(\bar{C})$.

Обозначим через $\varepsilon$ проекцию $Q \rightarrow B$, а через $v-$ проекцию $Q \rightarrow C$ и предположим, что $\varphi: \varepsilon \rightarrow \mu, \varphi: v \rightarrow \eta$. Обозначим $\bar{B}=\mu(\bar{Q}), \bar{C}=\operatorname{ker} \mu$. Тогда $\bar{C}-$ нормальная подлупа КЛМ $\bar{Q}$ и $\bar{Q}=\bar{B} \bar{C}$. Справедливо равенство $\varepsilon \varepsilon=\varepsilon$, поэтому и $\mu \mu=\mu$. Отсюда следует, что любой элемент, лежащий одновременно в $\mu \bar{B}$ и $\mu \bar{C}$, должен при $\mu$ переходить в себя и в 1 , так что $\bar{B} \cap \bar{C}=\{1\}$. Покажем, что $\bar{B}$ является нормальной подлупой КЛМ $\bar{Q}$. Пусть $b \in \bar{B}$ и пусть $u, v \in \bar{Q}$. Тогда $u=b_{1} c_{1}, v=b_{2} c_{2}$, где $b_{1}, b_{2} \in B, c_{1}, c_{2} \in C$. Далее,

$$
\begin{aligned}
\mu L(u, v) b & =\mu L^{-1}(u, v) L(u) L(v) b \\
& =L^{-1}(\mu u \cdot \mu v) L(\mu u) L(\mu v) \mu b \\
& =L^{-1}\left(\mu b_{1} \cdot \mu b_{2}\right) L\left(\mu b_{1}\right) L\left(\mu b_{2}\right) \mu b \in B .
\end{aligned}
$$

Следовательно, подлупа $\bar{B}$ нормальна в $\bar{Q}$, а тогда $\bar{Q}=\bar{B} \times \bar{C}$. Теперь, произведя отождествления

$$
\begin{array}{ll}
E(B)=\varepsilon E(Q) \varepsilon, & E(C)=v E(Q) v, \\
E(\bar{B})=\mu E(\bar{Q}) \mu, & E(\bar{C})=\eta E(\bar{Q}) \eta
\end{array}
$$


указанные в (b), из соотношений

$$
\varphi(\varepsilon E(Q) \varepsilon)=\mu E(\bar{Q}) \mu, \quad \varphi(\nu E(Q) v)=\eta E(\bar{Q}) \eta
$$

получаем, что изоморфизм $\varphi: E(Q) \rightarrow E(\bar{Q})$ индуцирует изоморфизмы

$$
E(B) \rightarrow E(\bar{B}), \quad E(C) \rightarrow E(\bar{C}) .
$$

Этим утверждение (с) доказано.

Следующее утверждение показывает, как изоморфизм двух сомножителей может быть определен в терминах эндоморфизмов.

(d) $\Pi y c m b$

$$
Q=B \times C=\bar{B} \times \bar{C}
$$

являются прямыми разложениями КЛМ $Q$ и $\varepsilon: Q \rightarrow B, \mu: Q \rightarrow \bar{B}-$ соответствуюшие проекции. Тогда $B \cong \bar{B}$, если и только если сущчествуют такие элементы $\alpha, \beta \in E(Q)$, что $\beta \alpha=\varepsilon и \alpha \beta=\mu$.

Если $\alpha, \beta \in E(Q)$ удовлетворяет этим равенствам, то из равенств

$$
\beta \alpha \beta=\varepsilon \beta=\beta \mu, \quad \alpha \beta \alpha=\alpha \varepsilon=\mu \alpha
$$

следует, что ограничение $\bar{\beta}$ гомоморфизма $\beta \alpha \beta$ на подлупу $B$ и ограничение $\bar{\alpha}$ гомоморфизма $\alpha \beta \alpha$ на подлупу $\bar{B}-$ это гомоморфизмы $\bar{\beta}: B \rightarrow \bar{B}$ и $\bar{\alpha}: \bar{B} \rightarrow B$. Далее, равенства

$$
(\beta \alpha \beta)(\alpha \beta \alpha)=\varepsilon, \quad(\alpha \beta \alpha)(\beta \alpha \beta)=\mu
$$

показывают, что $\bar{\alpha}$ и $\bar{\beta}-$ взаимно обратные отображения. Поэтому $B \cong \bar{B}$. Обратно, если $\bar{\beta}: B \rightarrow \bar{B}$ и $\bar{\alpha}: \bar{B} \rightarrow B-$ взаимно обратные изоморфизмы, то для $\beta=\varepsilon \bar{\beta}$ и $\alpha=\mu \bar{\alpha}$ справедливы равенства $\beta \alpha=\varepsilon$ и $\alpha \beta=\mu$.

(e) Легко проверить, что луповый изоморфизм $\alpha: Q \rightarrow B$ КЛМ индуцирует полугрупповой изоморфизм $\bar{\alpha}: E(Q) \rightarrow E(B)$, определяемый по формуле $\bar{\alpha}: \varphi \rightarrow \alpha \varphi \alpha^{-1}$. Перенесем этот факт на матричный язык в случае, когда КЛМ $Q$ обладает разложением (1) и $\alpha-$ автоморфизм КЛМ $Q$. Пусть $\alpha$ переводит разложение (1) в новое разложение

$$
Q=\bar{Q}_{1} \times \bar{Q}_{2} \times \ldots \times \bar{Q}_{r} \times \ldots,
$$

где $\bar{Q}_{i}=\alpha Q_{i}$, и пусть $\sigma-$ эндоморфизм КЛМ $Q$. Предположим, что эндоморфизму $\sigma$ в старом разложении соответствует $M, M$-матрица $\left(\sigma_{i k}\right)$ и в новом - матрица $\left(\varphi_{i k}\right)$. Для нахождения связи между этими матрицами надо учитывать следующее обстоятельство. Как указано перед теоремой 1, элементы матрицы зависят от конкретного прямого разложения и не являются самостоятельными объектами. В связи с этим введем следующее понятие.

Пусть $\omega-$ некоторый эндоморфизм КЛМ $Q$, пусть $Q_{1}$ - подлупа КЛМ $Q$ и пусть $Q_{2}=\omega Q_{1}$. Допустим далее, что задан некоторый изоморфизм $\beta$ между подлупами $Q_{1}, \bar{Q}_{1}$ и $Q_{2}, \bar{Q}_{2}$, а именно,

$$
\bar{Q}_{1}=\beta Q_{1}, \quad \bar{Q}_{2}=\beta Q_{2} .
$$

Тогда гомоморфизм $\omega: Q_{1} \rightarrow Q_{2}$ индуцирует гомоморфизм $\bar{\omega}: \bar{Q}_{1} \rightarrow \bar{Q}_{2}$, определяемый следующим образом. Если $\bar{a} \in \bar{Q}_{1}, \bar{a}=\beta a$, то полагаем

$$
\bar{\omega} \bar{a}=\bar{\omega} \beta a=\beta \bar{\omega} a=\overline{\omega a} .
$$


Теперь $M, M$-матрицу $\left(\sigma_{i k}\right)$, связанную с разложением (1), можно отнести и к разложению (3): каждому $\sigma_{i k}: Q_{k} \rightarrow Q_{i}$ сопоставляется соответствующее отображение $\varphi_{i k}: \alpha Q_{k} \rightarrow \alpha Q_{i}$. При этом матрице $\left(\varphi_{i k}\right)$ будет отвечать новый эндоморфизм $\varphi$ КЛМ $Q$. Найдем связь между $\varphi$ и $\sigma$. Пусть $a-$ произвольный элемент из $Q$. Тогда

$$
\begin{aligned}
\varphi \alpha a & =\sum_{k} \sum_{i} \varphi_{i k}(\alpha a)_{k}=\sum_{k} \sum_{i} \alpha \sigma_{i k} a_{k} \\
& =\alpha \sum_{k} \sum_{i} \sigma_{i k} a_{k}=\alpha \sigma a .
\end{aligned}
$$

Отсюда следует, что

$$
\varphi \alpha=\alpha \sigma, \quad \varphi=\alpha \sigma \alpha^{-1} \text {. }
$$

Если выбрать $M, M$-матрицу автоморфизма $\alpha$ по второму разложению, то мы придем к следующему утверждению: $M, M$-матрища $\left(\varphi_{i k}\right)$, соответствующая эндоморфизму $\sigma$ в старом разложение (1), и $M, M$-матрища $\left(\sigma_{i k}\right)$, соответствующая эндоморфизму $\varphi в$ новом разложении (3), сопряжены.

(f) Пусть теперь $Q-$ произвольная группа и $\Phi-$ ее группа автоморфизмов. Известно (см., например, [5]), что множество $\operatorname{Hol} Q$ пар $(\varphi, g), \varphi \in \Phi, g \in Q$, умножаемых по правилу

$$
(\varphi, g)\left(\varphi_{1}, g_{1}\right)=\left(\varphi \varphi_{1}, g \varphi_{1} \cdot g_{1}\right)
$$

образуют голоморф группы $Q$ (как и в [5] образ элемента $a$ при отображении $\varphi$ обозначим $a \varphi)$. Отображения

$$
\Phi \rightarrow \operatorname{Hol} Q, \quad Q \rightarrow \operatorname{Hol} Q,
$$

задаваемые по правилу $\varphi \rightarrow(\varphi, 1), g \rightarrow(1, g)$, являются изоморфными вложениями. Если отождествить $\Phi$ и $Q$ с подгруппами из $\operatorname{Hol} Q$ в силу этих вложений, то

$$
\varphi^{-1} g \varphi=g \varphi, \quad \varphi \in \Phi, \quad g \in Q,
$$

и $\mathrm{Hol} Q$ является полупрямым произведением $Q$ и $\Phi$. Равенство (5) показывает, что каждый автоморфизм $\varphi \in \Phi$ является сужением некоторого внутреннего автоморфизма группы $\mathrm{Hol} Q$.

Предположим теперь, что $Q$ является КЛМ и $\Phi$ - ее группа автоморфизмов. Проверяется, что множество $\operatorname{Hol} Q$ пар $(\varphi, g), \varphi \in \Phi, g \in Q$, умножаемых по правилу (4), образуют лупу и для нее справедливы предыдущие рассуждения, кроме последнего утверждения. Однако, если $\alpha, \beta \in \Phi$, то непосредственно проверяется, что внутренние подстановки $T(\alpha), L(\alpha, \beta), R(\alpha, \beta)$ являются автоморфизмами лупы Hol $Q$. Тогда равенство (5) показывает, что каждый автоморфизм $\varphi \in \Phi$ является сужением некоторой внутренней подстановки лупы Hol $Q$ вида $T(\varphi)$. Как и в случае групп, лупу Hol $Q$ назовем голоморфом КЛМ $Q$.

Далее приведем матричное представление голоморфа $\operatorname{Hol} Q$ КЛМ $Q$, обладающей прямым разложением (1), основанное на матричном представлении автоморфизмов КЛМ $Q$. Элементы из $Q$ будем рассматривать как строки, причем если $g=\sum_{i \in I} g_{i}$, то соответствующую строку запишем в виде $\bar{g}=\left(g_{1}, g_{2}, \ldots, g_{r}, \ldots\right)$. Согласно теореме 1 , группа Aut $Q$ может быть представлена $M, M$-матрицами вида $\left(\sigma_{i k}\right)$, где $\sigma_{i k}: Q_{k} \rightarrow Q_{i}$ - гомоморфизмы и любая подлупа $Q_{i}$ разложения (1) компонентно ассоциативна относительно 
своих подлуп $Q_{k} \sigma_{i k}$. Если $\varphi-$ автоморфизм из Aut $Q=\Phi$, то соответствующую $M, M$ матрицу обозначим через $\bar{\varphi}$. Строка $\bar{g}$ умножается на матрицу $\bar{\varphi}$ по обычному правилу матричного умножения:

$$
\begin{array}{r}
\bar{g} \bar{\varphi}=\left(g_{1} \varphi_{11}+g_{2} \varphi_{21}+\ldots+g_{r} \varphi_{r 1}+\cdots, g_{1} \varphi_{12}+g_{2} \varphi_{22}+\ldots+g_{r} \varphi_{r 2}+\cdots,\right. \\
\left.\ldots, g_{1} \varphi_{1 r}+g_{2} \varphi_{2 r}+\ldots+g_{r} \varphi_{r r}+\cdots, \ldots\right) .
\end{array}
$$

Здесь операция + - это умножение в подлупах $Q_{i}$ и она ассоциативна. В результате умножения получим строку, причем $\overline{g \varphi}=\bar{g} \cdot \bar{\varphi}$. Теперь рассмотрим $M, M$-матрицу вида

$$
M(\bar{\varphi}, \bar{g})=\left(\begin{array}{cc}
\bar{\sigma} & 0 \\
\bar{g} & 1,
\end{array}\right)
$$

где 0 - столбец из нулевых гомоморфизмов и 1 - единица КЛМ $Q$. Обозначим совокупность всех таких $M, M$-матриц через $\bar{M}$. Умножение таких матриц осуществляется по обычным правилам матричного умножения. С использованием клеточного умножения матриц непосредственно проверяется справедливость формул

$$
\begin{aligned}
M(\bar{\sigma}, \bar{a}) M(\bar{\varphi}, \bar{b}) & =M(\bar{\sigma} \bar{\varphi}, \bar{a} \bar{\varphi} \cdot \bar{b}), \\
M(E, \bar{a}) M(\bar{\sigma}, \overline{1}) & =M(\bar{\sigma}, \bar{a}), \\
M^{-1}(\bar{\sigma}, \overline{1}) M(E, \bar{a}) M(\bar{\sigma}, \overline{1}) & =M(E, \bar{a} \bar{\sigma}) .
\end{aligned}
$$

Здесь $E$ - единичная $M, M$-матрица (на главной диагонали единицы, а на остальных местах нули). Очевидно, что отображения $\Phi \rightarrow \bar{M}, Q \rightarrow \bar{M}$, задаваемые правилами

$$
\sigma \rightarrow\left(\begin{array}{cc}
\bar{\sigma} & 0 \\
\overline{1} & 1
\end{array}\right), \quad g \rightarrow\left(\begin{array}{cc}
E & 0 \\
\bar{g} & 1
\end{array}\right)
$$

являются изоморфными вложениями. Тогда совокупность $\bar{M}$ всех $M, M$-матриц вида (6) можно рассмотреть как матричное представление голоморфа КЛМ $Q$, обладающая прямым разложением (1).

\section{Список литературы}

1. Плоткин Б. И., Группы автоморфизмов алгебраических систем. Наука, Москва, 1966.

2. Bruck R. H., A survey of binary systems. Springer, Berlin, 1958.

3. Санду Н. И., О центрально нильпотентных коммутативных лупах Муфанг. В сб.: Квазигруппь $u$ лупы. Штиинца, Кишинев, 1979, с. 145-155.

4. Фукс Л., Бесконечные абелевы группы, 2. Мир, Москва, 1977.

5. Каргаполов М. И., Мерзляков Ю. И., Основы теории групn. Наука, Москва, 1977.

Статья поступила 8.11.2007. 